\title{
Cognitive and Memory Improvement by Duloxetine Administration in Demented Adult APP/PS1 Transgenic AD Mouse Model
}

\section{Sahar Mohamed Kamal Shams El Dine*}

Department of Pharmacology, Faculty of Medicine, University of Ain-Shams, Cairo, Egypt

\begin{abstract}
Alzheimer's disease (AD) are often associated with memory and cognitive deficits. Effective treatment of these diseases leads to a marked improvement in the cognitive function of such patients.

There is a suggestion that there is some neuroprotective properties of duloxetine, as one of the antidepressants, against dementia-associated with cognitive disorders. The present study assessed cognitively enhancing property of duloxetine in a demented adult APP/PS1 transgenic mouse model of AD. Intraperitoneal (ip) administration of a single dose of duloxetine $10 \mathrm{mg} / \mathrm{kg} /$ day before each test. This drug treatment rescued cognitive deficits in APP/PS1 mice in both cognitive tests used in this study. The results of the present study suggest that duloxetine administration can help in improvement of cognitive disorder and may inhibit any memory impairement that accompanied the pathogenesis of AD.
\end{abstract}

Keywords: Alzheimer's disease; Mouse; Duloxetine; Intraperitoneal; Cognitive

\section{Introduction}

Cognitive impairment should be considered as an important sign as other emotional and physical symptoms in patients with many mental diseases [1]. Duloxetine hydrochloride is a drug that was approved to be an antidepressant medication via inhibition of both serotonin (5HT) and norepinephrine reuptake [2]. An interesting idea about the possible dual beneficial effect of antidepressant drugs in the treatment of depression with cognitive impairment via the correction of the imbalance or deficiency in 5-HT and/or norepinephrine systems was created as a hypothesis in the last century by many researchers related to improvement of associated cognitive deficits with major depression [3]. Duloxetine is an antidepressant drug that researchers try all the time to prove its "dual-action" as both antidepressant and a drug with cognitive and memory improvement "inootropic drug". However, no recent experimental studies have specifically focused on the possible cognitive and memory improvement of duloxetine in the treatment of depressed mood [4,5].

Because the effect of duloxetine on cognitive performance who commonly suffer from mental disorders has not markedly evaluated, the primary objective of the present study was to assess the cognitive and memory efficacy of duloxetine, that was administered in a dose of $10 \mathrm{mg} /$ day ip before each test in a demented adult APP/PS1 transgenic mouse model of AD.

\section{Material and Methods}

\section{Animals}

Demented adult APP/PS1 transgenic AD mouse model 25-30 gm (3 months old) was purchased from Animal House of Abou-El-Reech animal Institute, Giza, Egypt. They were stored kept in a temperature $\left(22 \sim 24^{\circ} \mathrm{C}\right)$ and humidity $(50 \sim 60 \%)$ controlled central animal house facility under light $(12 \mathrm{~h})$ and dark (12 h) illumination cycle. Animals were given free access to standard food and water. Experiments were performed between 12.00-16.00 h. Each mouse was exposed to elevated plus maze (EPM) and Morris water maze (MWM). In each set, animals were randomly distributed into 2 groups ( $\mathrm{n}=6 /$ group). The arena of EPM was cleaned using $70 \%$ ethyl alcohol solution before placing each mouse.

\section{Ethics}

Experimental protocols used in present were approved by the Institutional Animal Ethics Committee, Faculty of Medicine, Ain shams university, Cairo, Egypt.

\section{Duloxetine treatment and animal grouping}

Duloxetine hydrochloride (Sigma Chemical Co, USA), wase administered through intra-peritoneal route (ip). Normal saline $(0.9 \%$ $\mathrm{w} / \mathrm{v} \mathrm{NaCl}$ ) was used to prepare drug solution. Each mouse received treatment $1 \mathrm{~h}$ before test session in EPM (on 2nd day) and Morris water maze (MWM- on 6th day). There were 2 groups of in a demented adult APP/PS1 transgenic mouse model of $\mathrm{AD}$ mice:

Control group (Group I): As in a demented adult APP/PS1 transgenic mouse model of $\mathrm{AD}$, received normal saline $(10 \mathrm{ml} / \mathrm{kg})$ Treated-group of a demented adult APP/PS1 transgenic mouse model of $\mathrm{AD}$ (Group 2) was administered with duloxetine $(10 \mathrm{mg} / \mathrm{kg})$.

\section{Spatial memory tests}

EPM: The protocol accustomed appraise to transfer latency (TL) in EPM was delineate by Dhingra et al., [6]. In brief, a pair of open arms $(30 \times 5 \mathrm{~cm})$ and a couple of closed arms $(30 \times 5 \times 12 \mathrm{~cm})$ of EPM were organized in order that the two closed arms unbroken opposite to every alternative with associate degree open roof. Every animal was placed at the top of open arm facing faraway from central platform $(5 \times 5 \mathrm{~cm})$. On the primary day (the acquisition session), every animal was exposed to EPM for ninety seconds. Time taken by animal to succeed in the

*Corresponding author: El Dine SMKS, Department of Pharmacology, Faculty of Medicine, University of Ain-Shams, Cairo, Egypt; Tel: 0020224186992; E-mail: saharkamal2003@hotmail.com

Received March 04, 2015; Accepted May 13, 2015; Published May 25, 2015

Citation: EI Dine SMKS (2015) Cognitive and Memory Improvement by Duloxetine Administration in Demented Adult APP/PS1 Transgenic AD Mouse Model. J Nanomed Nanotechnol 6: 296. doi:10.4172/2157-7439.1000296

Copyright: ( 92015 El Dine SMKS. This is an open-access article distributed under the terms of the Creative Commons Attribution License, which permits unrestricted use, distribution, and reproduction in any medium, provided the original author and source are credited. 
closed arm was recorded because the transfer latency (TL). Animals didn't enter in closed arm in ninety seconds were excluded from study. On second day (the retention session), every animal was place into the open arm and also the atomic number 81 was recorded for optimum ninety seconds. The good v2.5.21 video- chase system (Panlab Harvard equipment, Spain purchased from Al-Amyria instrument company, Giza, Egypt) was accustomed.

Morris water maze (MWM): MWM check is employed to judge the hippocampal-dependent learning, together with acquisition of abstraction memory and long abstraction memory. The protocol of MWM delineated by Bromley- Brits et al., [7] was wont to confirm the p.c time spent in target quadrant. Drug treatments got to mice sixty min before check on sixth trial day. In brief, the pool having a hundred and fifty $\mathrm{cm}$ diameter and, fifty $\mathrm{cm}$ depth was made of seamless black polythene. The clear plastic escape platform $(10 \mathrm{~cm}$ diameter, thirty one $\mathrm{cm}$ high) may well be positioned within the any one of four quadrant position within the pool. The water temperature was maintained at temperature $\left(22 \sim 24^{\circ} \mathrm{C}\right)$. Every animal went through coaching trials (5 trials each day) from day one to day five. On 1st day, platform was visible $(1 \mathrm{~cm}$ higher than water level) and placed in south-west, north-west, north- east, centre, and south-west positions in five trials, severally. Beginning directions of animal in five trials were south (S), north $(\mathrm{N}), \mathrm{S}$, east (E), and west (W), severally. On 2 5th days, platform was created hidden (at water level) and unbroken in S-W position. The beginning locations of every animal in five trials were W-S-N-E-S (2nd day), N-E-W-W-S (3rd day), N-E-W-S-N (4th day), and E-S-W-E-N (5th day). On the sixth day, just one trial was performed having $\mathrm{N}$ as beginning location of animal and while not platform. The time spent in target quadrant (SW) was noted as index of retrieval or memory. Video camera was mounted on the ceiling to record the behavior of the mice within the pool.

\section{Statistical analysis}

The Graphpad Prism Windows version 5 was used to perform statistical analysis. Comparison between different groups was performed using ANOVA followed by Tukey's test to determine the significant difference as a post-hoc test. Data was represented as mean $\pm S D$ values (per group $n=6 /$ group). The level of significance was $p<0.05$.

\section{Results}

Figure 1 showed the period of Elevated plus maze - Transfer Latency (TL) in both tested groups. There was a marked reduction in $\mathrm{TL}$ in duloxetine-treated group as mean $\pm \mathrm{SD}(80 \pm 6.5 \mathrm{sec}$. in control group versus $34.17 \pm 4.8 \mathrm{sec}$. in duloxetine-treated group). There is a percentage reduction by $57.29 \mathrm{sec}$ in TL by duloxetine treatment in such transgenic model.

Figure 2, Morris water maze. (A) Escape latency (visible platform) measured on 1st day. (B) Escape latency (invisible platform) measured between 2 nd and 5 th days. (C) Time spent in target quadrant on 6th day. Data is presented as mean $\pm \mathrm{SD}$ ( $\mathrm{n}=6$ /group).

There was a marked reduction in any delay induced in the control group compared to duloxetine-treated group as mean \pm SD (51.61 \pm $2.7 \mathrm{sec}$. in control group versus $19.94 \pm 2.0 \mathrm{sec}$. in duloxetine-treated group). There is a percentage reduction by $61.36 \mathrm{sec}$ in any delay in movement by duloxetine treatment in such transgenic model.

\section{Discussion}

The results of the present study suggest that duloxetine administration can help in improvement of cognitive disorder and may
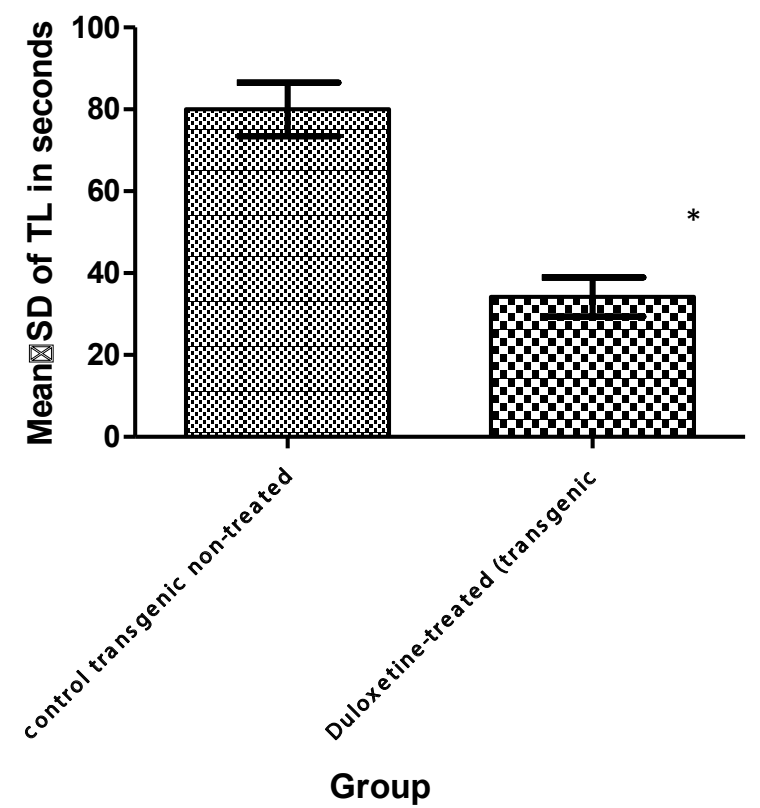

Figure 1: ${ }^{*} p<0.05$ as a significant difference - as compared to the control group ( $n=6 /$ group).

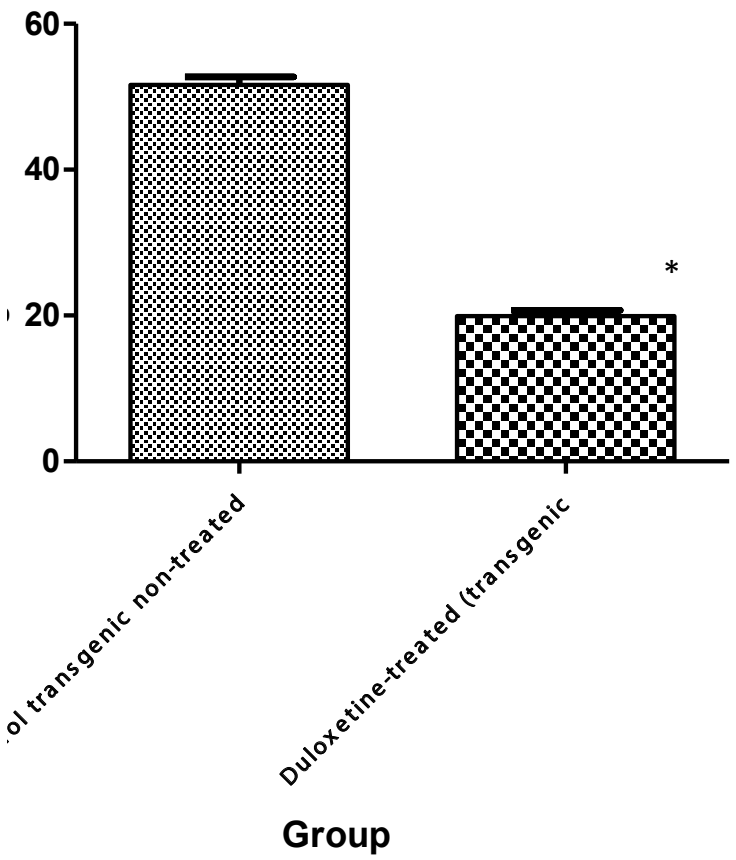

Figure 2: ${ }^{*} p<0.05$ represents significant difference - as compared against the control group. 
Citation: EI Dine SMKS (2015) Cognitive and Memory Improvement by Duloxetine Administration in Demented Adult APP/PS1 Transgenic AD Mouse Model. J Nanomed Nanotechnol 6: 296. doi:10.4172/2157-7439.1000296

Page 3 of 3

inhibit any memory impairement that accompanied the pathogenesis of $\mathrm{AD}$, after its administration in a dose of $10 \mathrm{mg} / \mathrm{kg}$ ip in a demented adult APP/PS1 transgenic mouse model of AD.

Fuller et al., [8] revealed that duloxetine, (+)-N-methyl-3-(1naphthalenyloxy)-2-thiophenepropanamine, is an inhibitor of the serotonin and norepinephrine neuronal transporters. In their tested mice, duloxetine antagonized the depletion of brain serotonin by p-chloroamphetamine (ED50 $=2.5 \mathrm{mg} / \mathrm{kg}$, i.p.) and the depletion of heart norepinephrine by 6-hydroxydopamine (ED50 $=1.1 \mathrm{mg} / \mathrm{kg}$, i.p.). Brain concentrations of 5-hydroxyindoleacetic acid were decreased by duloxetine at $2 \mathrm{hr}$ after doses of 1,3 and $10 \mathrm{mg} / \mathrm{kg}$ and at 1 to $8 \mathrm{hr}$ (but not $24 \mathrm{hr}$ ) after a $10 \mathrm{mg} / \mathrm{kg}$ i.p. dose of duloxetine. Duloxetine antagonized monoamine neurotransmitter depletion in frontal area, however not monoamine neurotransmitter depletion in corpus striatum, once treatment of mice with 1-methyl-4-phenyl-1,2,3,6-tetrahydropyridine. In rats, duloxetine cut brain 5-hydroxyindoleacetic acid dosedependently for up to eight 60 minutes and cut 5-hydroxytryptamine turnover measured by the buildup of 5-hydroxytryptophan in rat neural structure once enzyme inhibition. In rats, duloxetine antagonized the depletion of brain 5-hydroxytryptamine by p-chloramphetamine and also the depletion of monoamine neurotransmitter and endocrine in neural structure once the injection of 6-hydroxydopamine. In vitro, duloxetine had very little result on either blood type (serotonin as substrate) or blood type (phenylethylamine as substrate) enzyme, IC50 concentrations being higher than $10(-5) \mathrm{M}$.

The reduction in escape latency observed from the first day to 5 th day as shown in Figure 2, is supported by clinical studies that have reported cognition related benefits with 8 weeks [9] and 12 weeks [10] of duloxetine treatment in depressed patients. Many published reports showed that there is a significant increase in brain monoamine profile of duloxetine including norepinephrine, serotonin and other chemical neurotransmitters $[11,12]$. These reports suggest also that duloxetine increases dopamine (DA) levels not only in cerebral cortex $[11,12]$ but also in hippocampus [13] and nucleus accumbens region [12]. Frontal cortex and hippocampus regions play important role in cognition and emotions [14]. The possible reason behind the ability of duloxetine to produce augmentation of nootropic activity that appears in the marked cognitive and memory functions in this model of transgenic mice may be the interactions between the increase in serotonin and norepinephrine and duloxetine in the important brain's area for both functions, the hippocampus [10]. Older generation of antidepressants as tricyclic antidepressants, amitriptyline showed a reduction in memory and learning tasks due to a low action of amitriptyline serotonin and norepinephrine re-uptake as suggested by Everss et al., [15].

\section{Conclusion}

Further studies that would focus on the effect of acute and chronic dosing of duloxetine on electrophysiological analysis, neurogenesis, biogenic amine pathway activation/deactivation, drug metabolism, and related drug interaction studies may help in supporting the results of the present study outcomes that would benefit the patients with AD.

\section{Disclosure}

The author reports no conflicts of interest in this work.

\section{Acknowledgment}

This research was officially supported by the Medical Research Service of the Ain Shams University. It was financially supported by the laboratory of the Pharmacology Department, Faculty of Medicine, Ain Shams University.

\section{References}

1. Schaffer S, Takahashi K, Azuma J (2000) Role of osmoregulation in the actions of taurine. Amino Acids 19: 527-546.

2. Su JH, Anderson AJ, Cummings BJ, Cotman CW (1994) Immunohistochemical evidence for apoptosis in Alzheimer's disease. Neuroreport 5: 2529-2533.

3. Matsuyama Y, Morita T, Higuchi M, Tsujii T (1983) The effect of taurine administration on patients with acute hepatitis. Prog Clin Biol Res 125: 461 468.

4. Raskin J, Wiltse CG, Siegal A, Sheikh J, Xu J, et al. (2007) Efficacy of duloxetine on cognition, depression, and pain in elderly patients with major depressive disorder: an 8-week, double-blind, placebo-controlled trial. Am J Psychiatry 164: 900-909.

5. Centers for Disease Control and Prevention, Gangadharan D, Smith J Weyant R (2013) Biosafety Recommendations for Work with Influenza Viruses Containing a Hemagglutinin from the A/goose/Guangdong/1/96 Lineage. MMWR Recomm Rep 62: 1-7.

6. Dhingra D, Parle M, Kulkarni SK (2004) Memory enhancing activity of Glycyrrhiza glabra in mice. J Ethnopharmacol 91: 361-365.

7. Bromley-Brits K, Deng Y, Song W (2011) Morris water maze test for learning and memory deficits in Alzheimer's disease model mice. J Vis Exp.

8. Fuller RW, Hemrick-Luecke SK, Snoddy HD (1994) Effects of duloxetine, an antidepressant drug candidate, on concentrations of monoamines and their metabolites in rats and mice. J Pharmacol Exp Ther 269: 132-136.

9. http://www.webmd.com/drugs/2/drug-91490/duloxetine-oral/details

10. Greer TL, Sunderajan P, Grannemann BD, Kurian BT, Trivedi MH (2014) Does duloxetine improve cognitive function independently of its antidepressant effect in patients with major depressive disorder and subjective reports of cognitive dysfunction. Depress Res Treat: 627-863.

11. Kihara T, Ikeda M (1995) Effects of duloxetine, a new serotonin and norepinephrine uptake inhibitor, on extracellular monoamine levels in rat frontal cortex. J Pharmacol Exp Ther 272: 177-183.

12. Muneoka K, Shirayama Y, Takigawa M, Shioda S (2009) Brain region-specific effects of short-term treatment with duloxetine, venlafaxine, milnacipran and sertraline on monoamine metabolism in rats. Neurochem Res 34: 542-555.

13. Kale PP, Addepalli V (2014) Augmentation of antidepressant effects of duloxetine and bupropion by caffeine in mice. Pharmacol Biochem Behav 124: 238-244.

14. Eriksson TM, Delagrange P, Spedding M, Popoli M, Mathé AA, et al. (2012) Emotional memory impairments in a genetic rat model of depression: involvement of 5-HT/MEK/Arc signaling in restoration. Mol Psychiatry 17: 173184.

15. Everss E, Arenas MC, Vinader-Caerols C, Monleón S, Parra A (2005) Piracetam counteracts the effects of amitriptyline on inhibitory avoidance in CD1 mice. Behav Brain Res 159: 235-242. 